\title{
Fungal Infective Endocarditis mimicking Atrial Myxoma: Transesophageal Echocardiography Assessment
}

\author{
${ }^{1}$ Neeti Makhija, ${ }^{2}$ Kalpna Irpachi, ${ }^{3}$ Palleti Rajashekar, ${ }^{4} \mathrm{Ch}$ Bharat Siddharth
}

\begin{abstract}
Infective endocarditis (IE) accounts for 0.5 to 1 of every 1,000 hospital admissions. This case describes a left atrial mass of fungal etiology mimicking an atrial myxoma. At times, the diagnosis of mass in left atrium can be a challenge, which is discussed in this report.
\end{abstract}

Keywords: Infective endocarditis, Myxoma, Transesophageal echocardiography.

How to cite this article: Makhija N, Irpachi K, Rajashekar P, Siddharth CB. Fungal Infective Endocarditis mimicking Atrial Myxoma: Transesophageal Echocardiography Assessment. J Perioper Echocardiogr 2017;5(2):82-85.

\section{Source of support: Nil}

Conflict of interest: None

\section{INTRODUCTION}

Cardiac masses often present a clinical challenge in their differential diagnosis, and in many cases only biopsy can confirm the final diagnosis. A left atrial mass attached to cardiac structures can be either a primary tumor, commonest being myxoma, a thrombus, or a vegetation. Infective endocarditis accounts for 0.5 to 1 of every 1,000 hospital admissions. ${ }^{1}$ We present a case of IE of fungal etiology mimicking left atrial myxoma.

\section{CASE REPORT}

A 26-year-old pregnant woman presented to the labor ward with a history of multiple abortions with no known comorbidities. She underwent emergency lower segment cesarean section and was transferred to the postnatal ward. On first postoperative day, she had developed anemia, anuria, abdominal distension, and respiratory distress. Investigations revealed low platelets and deranged renal

\footnotetext{
${ }^{1}$ Professor, ${ }^{2,4}$ Senior Resident, ${ }^{3}$ Associate Professor

${ }^{1,2}$ Department of Cardiac Anesthesia, All India Institute of Medical Sciences, New Delhi, India

${ }^{3,4}$ Department of Cardiothoracic and Vascular Surgery, All India Institute of Medical Sciences, New Delhi, India

Corresponding Author: Neeti Makhija, Professor, Department of Cardiac Anesthesia, All India Institute of Medical Sciences New Delhi, India, Phone: +911126593423, e-mail: neetimakhija@ hotmail.com
}

parameters. After a few days, she was electively intubated and started on alternate day hemodialysis. She developed high-grade fever despite higher generation antibiotics. Repeat blood cultures for bacteria and fungus were sterile. Subsequently, transthoracic echocardiography (TTE) was done, which was normal. However, dengue serology was positive and the patient was managed symptomatically. Later, the patient was extubated and started orally, but high-grade fever and anuria persisted with increasing serum creatinine. Renal biopsy was done which showed renal cortical necrosis. She also underwent intravitreal injection for endophthalmitis.

After a few days, she again developed sudden-onset respiratory distress as well as tachycardia with features of congestive cardiac failure. Repeat TTE revealed a large mass on posterior mitral leaflet (PML) with severe mitral regurgitation. In view of the clinical course of illness, and presence of mass on TTE, IE was suspected. Serum galactomannan was highly positive $(>6$; normal $<0.5)$, suggesting fungal infection. Amphotericin B was started. In view of progressive deterioration of the hemodynamics and large size of vegetation, she was taken up for mitral valve (MV) replacement surgery.

Intraoperatively, transesophageal echocardiography (TEE) examination using X7-2t Philips ultrasound probe and ultrasound machine (Phillips iE33 model, Bothell, Washington, USA) was performed. Two-dimensional (2D) midesophageal (ME) four-chamber view at sector angle of $0^{\circ}$ (Fig. 1) and three-dimensional (3D) image

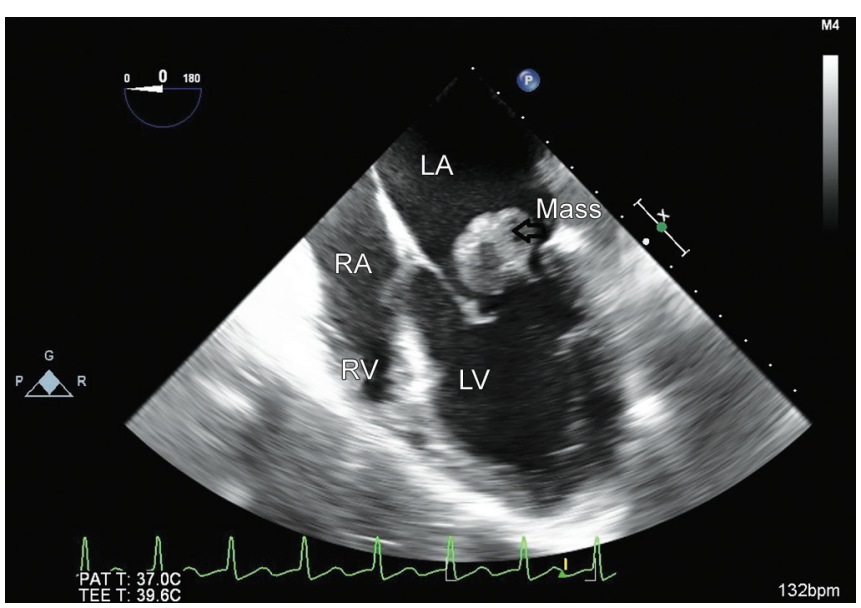

Fig. 1: A 2D mid-esophageal four-chamber view at sector angle of $0^{\circ}$ showing a mass in the LA 


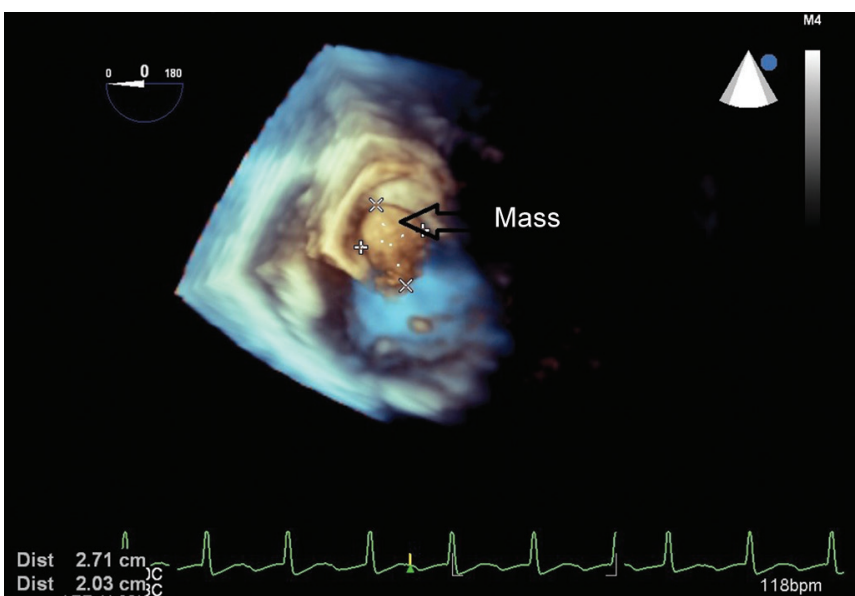

Fig. 2: A 3D mid-esophageal image of left atrial mass with measurements

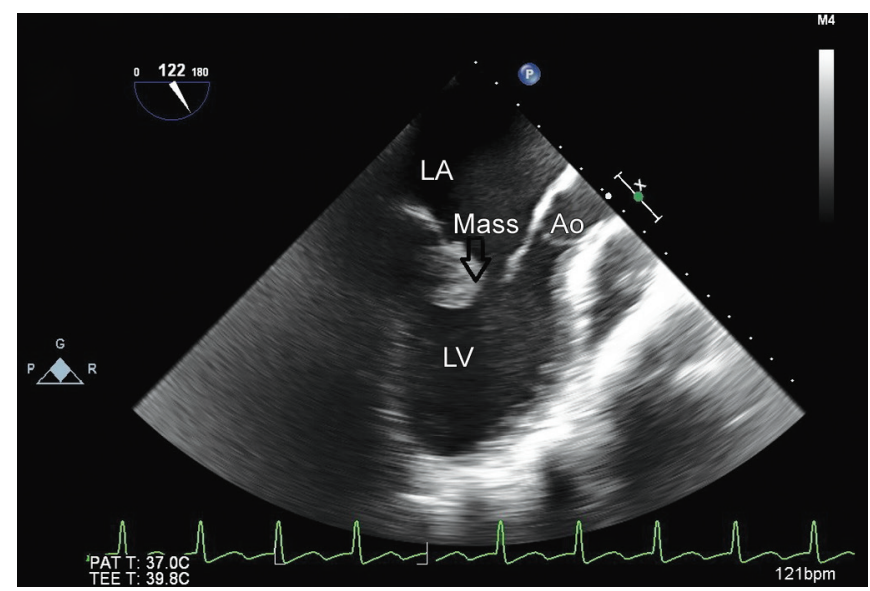

Fig. 4: A 2D mid-esophageal long-axis view at sector angle of $122^{\circ}$ showing a mass attached to PML

(Fig. 2) revealed a large mass of $2.7 \times 2 \mathrm{~cm}$ attached to the PML. A continuous wave Doppler in the 2D ME four-chamber view across MV along with intervening mass had mean and maximum gradients of 5 and 16 $\mathrm{mm} \mathrm{Hg}$ respectively. The color flow Doppler across MV in the same view revealed mitral regurgitation (Fig. 3 and Video 1). A 2D ME aortic valve long-axis view at sector angle of $122^{\circ}$ (Fig. 4 and Video 2) and 2D deep transgastric (TG) long-axis view (Fig. 5 and Video 3) at sector angle of $0^{\circ}$ confirmed that the mass was mobile, with heterogeneous echogenicity. The mass was attached to the PML by a stalk. A 3D deep TG long-axis view also showed a mass attached to the PML, but was now seen clearly to be consisting of multiple small masses and thread-like structures (Fig. 6 and Video 4). Peroperatively, there was destruction of the PML. Multiple small masses adherent to each other were present on the PML and a few extending onto the chordae. Mitral valve replacement was done using 25/33 ON-X mechanical valve. Postoperatively on TEE, prosthetic valve function was normal. Patient was electively ventilated for

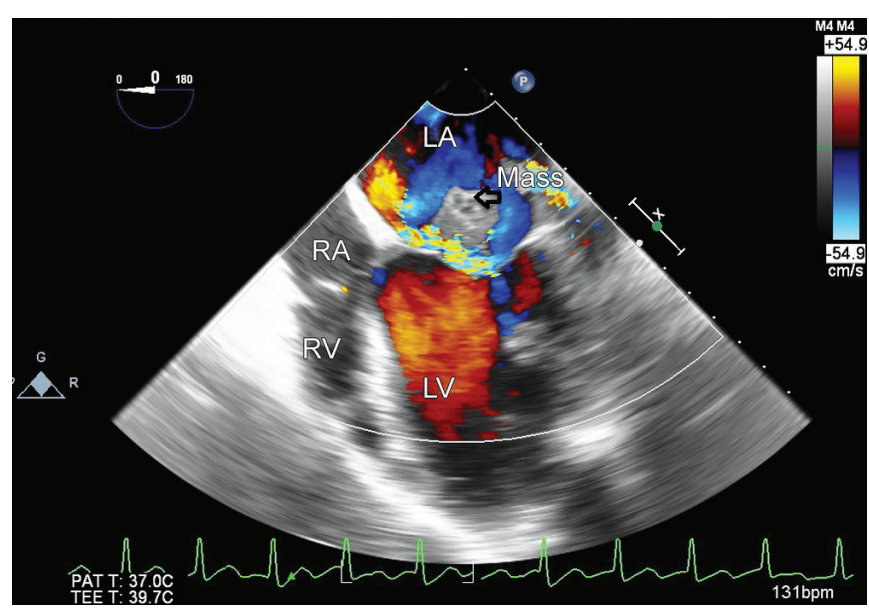

Fig. 3: A color flow Doppler across MV in the 2D mid-esophageal four-chamber view at sector angle of $0^{\circ}$ showing mitral regurgitation

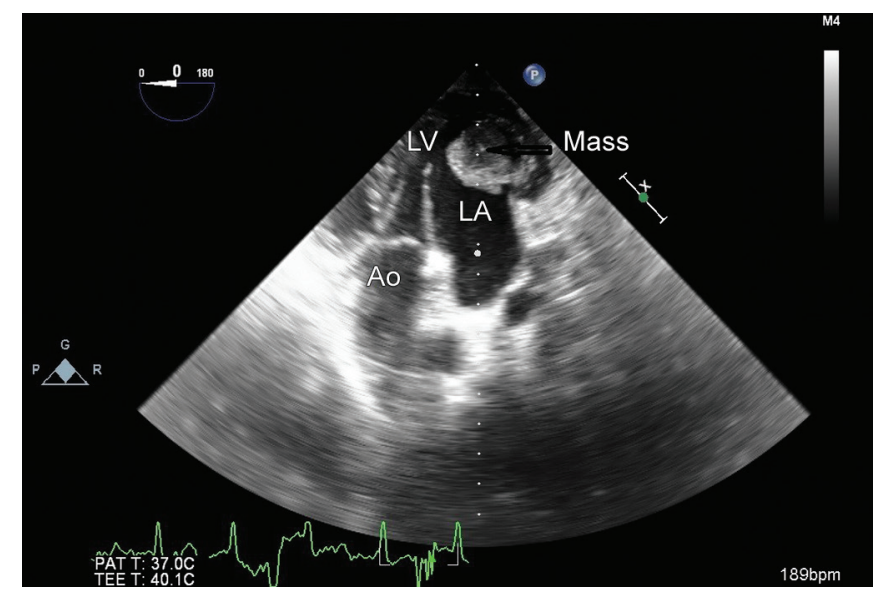

Fig. 5: A 2D deep TG long-axis view at sector angle of $0^{\circ}$ showing a mass attached to PML

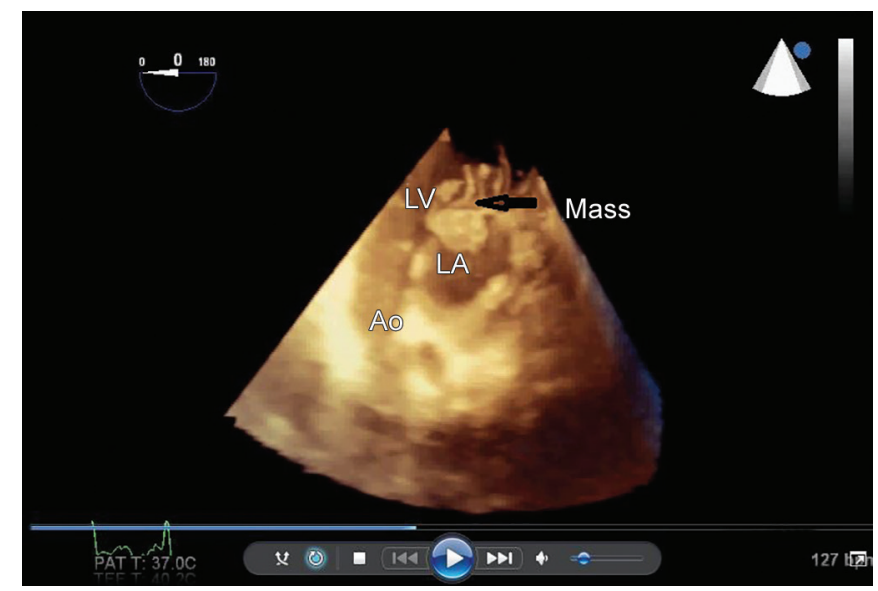

Fig. 6: A 3D deep TG long-axis view at sector angle of $0^{\circ}$ showing a mass attached to PML

24 hours, thereafter, weaned off inotropes and extubated on third day. In view of decreased neurological response, noncontrast computerized tomography head was done, which revealed multiple chronic infarcts, probably due to systemic embolization. Histopathology of valve tissue showed growth of aspergillus. Intravenous 
voriconazole was started. Patient remained on continuous hemodialysis as renal function did not improve. Finally, she succumbed to severe fungal sepsis on the tenth postoperative day.

\section{DISCUSSION}

Myxomas are the most common primary cardiac tumors of the left atrium. They may simulate IE but are rarely infected. Vogt et $\mathrm{al}^{2}$ presented a patient with infected left atrial myxoma combined with MV endocarditis, treated by excision of the myxoma and MV reconstruction.

Roberts $^{3}$ reported four patients with diagnosis of thickened MV with degeneration of the cusps, left atrial myxoma, right atrial myxoma, and organized thrombus in the left atrium, with mitral stenosis that mimicked active bacterial endocarditis of the MV. The cases were obtained from a series of $130 \mathrm{MV}$ operations performed at the National Heart Hospital, London, in a 3-year period.

On the contrary, IE can also mimic myxoma as in our patient. von Kemp et $\mathrm{al}^{4}$ have also reported a patient with group II streptococcal endocarditis and large vegetation resembling MV myxoma.

Infective endocarditis usually involves one or more heart valves resulting in vegetation, perforation, abscess, fistula, and/or pseudoaneurysm formation. In IE, the mainstay of treatment remains antimicrobial agent for 4 to 6 weeks. However, 40 to $60 \%$ require surgical intervention because of some complications. Surgery could be risky in the active stage of the disease, but it becomes essential to avoid preoperative heart failure, irreversible structural damage, and systemic embolization. ${ }^{5}$ These cases carry a higher risk of perioperative morbidity.

Echocardiographic features of myxoma are globular, smooth-surfaced, or irregular, friable surface with multilobular appearance, 4 to $8 \mathrm{~cm}$ in size, heterogeneous echogenicity with areas of echolucency, and sometimes calcifications. ${ }^{6}$

Echocardiographic features typical for endocarditis are masses attached primarily to a valve rather than myocardium, smaller size of mass/vegetation $(<3 \mathrm{~cm})$, and long strand-like appearance. In addition, there is a typical clinical picture, organism on blood cultures, and resolution with successful antimicrobial therapy. Often the only way to distinguish atrial myxoma from IE is by pathological examination of myxoma tissue retrieved from a peripheral artery or from cardiac surgery. The rare case with left atrial IE and giant vegetation mimicking left atrial myxoma is reported in the literature. ${ }^{7}$

Transesophageal echocardiography should describe the size, shape, mobility, number, and location of the vegetation. Vegetations are common on the low-pressure side of the regurgitation valve, i.e., ventricular side of the aortic valve or left atrial aspect of the MV. The other characteristic feature of vegetations is that their motion is chaotic and independent of cardiac cycle. At times, they may be found in the chamber wall and their sizes vary from 0.1 to $1 \mathrm{~cm}$, which is sometimes confused with left atrial masses. Thus, TEE helps in the detection of site of attachment of left atrial masses, as well as in postoperative evaluation. Papadopoulos et $\mathrm{al}^{8}$ demonstrated the added value of $3 \mathrm{D}$ TEE in the differential diagnosis of the cardiac masses.

Aspergillus endocarditis is generally considered fatal because it is difficult to diagnose and treat, which typically occurs in severely immunosuppressed patients. Walsh and Hutchins ${ }^{9}$ found a $40 \%$ prevalence of aspergillosis with cardiac involvement.

In our patient, the appearance of mass on TEE was consistent, with left atrial myxoma being a mobile heterogeneous mass with areas of echolucency. Attachment of mass onto MV was consistent with both a myxoma and IE. ${ }^{5}$ It was only with the help of 3D TEE, that too in deep TG long-axis view, that the mass could be seen to be consisting of multiple masses and strands-like structure attached to PML, which was consistent with IE. Histological examination confirmed aspergillosis endocarditis with no evidence of myxoma. Clinical features were also in favor of IE.

\section{CONCLUSION}

So, to conclude, an echocardiographer should confirm the diagnosis of a left atrial mass only after assessment in all the views, including $3 \mathrm{D}$ views. Also, the clinical setting should be taken into consideration. Infective endocarditis of the MV may mimic the atrial myxoma despite the fact that TEE remains the gold standard for noninvasive assessment of intracardiac structures.

\section{REFERENCES}

1. Dajani, A.; Taubert, K. Infective endocarditis. In: Allen HD, Gutgesell HP, Clark EB, Driscoll DJ, editors. Moss and Adams' heart disease in infants, children and adolescents. Philadelphia (PA): Lippincott Williams \& Wilkins; 2001. pp. 1297-1308.

2. Vogt PR, Jenni R, Turina MI. Infected left atrial myxoma with concomitant mitral valve endocarditis. Eur J Cardiothorac Surg 1996 Jan;10(1):71-73.

3. Roberts N. Mimics of bacterial endocarditis. Am J Cardiol 1970 Nov;26(5):528-531.

4. von Kemp K, Demoor D, Caes F, Cham B, Block P. Group B streptococcal endocarditis mimicking mitral valve myxoma. Acta Cardiol 1987;42(3):223-228.

5. Kiran U,Chowdhury A,Makhija N, Saini K, Rajput RS, Airan B. Infective endocarditis: the anesthesiologists perspective. J Perioper Echocardiogr 2013 Jul-Dec;1(2):57-61. 
6. Makhija N, Irpachi K, Chowdhury UK, Kiran U. Unusual attachment of left atrial myxoma: role of transesophageal echocardiography. J Perioper Echocardiogr 2016 Jan-Jun; 4(1):30-33.

7. Bayram NA, Ayhan H, Keles T, Durmaz T, Bozkurt E. Infective endocarditis mimicking left atrial myxoma. J Echocardiogr 2010 Dec;8(4):124-125.
8. Papadopoulos CH, Michalakeas CA, Paraskevaidis I, Ikonomidis I, Anastasiou-Nana M. Differential diagnosis of a left atrial mass: role of three-dimensional transoesophageal echocardiography. Hellenic J Cardiol 2010 Nov-Dec;51(6): 546-548.

9. Walsh TJ, Hutchins GM. Aspergillus mural endocarditis. Am J Clin Pathol 1979 Jun;71(6):640-644. 